\title{
How to Efficiently and Effectively Supervise Drug Distribution in China?
}

\author{
Manman Zhang ${ }^{\mathrm{a}}$, Yi Liu ${ }^{\mathrm{b}}$, Tianhua Zhang ${ }^{\mathrm{c}}$ \\ Department of Logistics Management, School of Economics and Management, Beijing Jiaotong \\ University, Beijing, 100044, China \\ amanmanzhang@bjtu.edu.cn, byliu@bjtu.edu.cn, ${ }^{\mathrm{c}} 13113143 @$ bjtu.edu.cn,
}

Keywords: Regulation, healthcare policy, drug supervision.

\begin{abstract}
Objective: Many incidents related to fake/inferior-quality drugs have occurred in China recently. The China Food and Drug Administration (CFDA) faces the challenge of efficiently and effectively supervising drug distribution to prevent fake/inferior-quality drugs from entering the drug market. The aim of this research is to help CFDA find methods to efficiently and effectively supervising drug distribution.
\end{abstract}

Method: We analyze the law base and supervision methods adopted by CFDA and find the root causes of inefficient and ineffective supervision of drug distribution in China. Then we propose adopting a classified supervision approach, whereby we suggest a new classification method based on the safety requirements and economic values of drugs, and deploy a proper supervision approach for each class of drugs.

Results and Conclusions: The main causes of frequent occurrence of incidents related to fake/inferior-quality drugs is the supervision method adopted by CFDA. Because of the tussle between the increasing need for drug supervision and the limit of drug supervision resources, CFDA should adopt classified supervision approach and deploy different supervision method for different class of drugs.

\section{Introduction}

Drugs are special commodities that must meet the quality standards set by governments to ensure their safe and effective use for treating patients. Fake and inferior-quality drugs are harmful to patients and society. However, drugs are generally high-tech and experience products, the ingredients and functions of which cannot be easily identified by the patients themselves. So governments must closely supervise drug manufacturing, sale, and distribution to prevent fake or inferior-quality drugs from entering the drug market.

Due to the unsubstitutability, indispensability and high profitability of many drugs, a large number of incidents related to fake or inferior-quality drugs have occurred in China in recent years. The China Food and Drug Administration (CFDA) reported that 89,226 drug criminal cases were found in China in $2015^{[1]}$, in which the total pharmaceutical value was about RMB $\$ 540$ million, and 96,825 cases and RMB $\$ 633$ million pharmaceutical value in $2016^{[2]}$. Recently, a peasant in Ninghai County, Zhejiang Province, employed ten persons to produce 90,000,000 poisonous capsules by using industrial gelatin. While the offender was arrested on 3 September 2014, where the poisonous capsules had been dispatched could not be found out yet. Such drug-related incidents bring great health risks to patients and have an adverse effect on society ${ }^{[3]}$.

The difficulty of supervising drug distribution stems from the tussle between the increasing need for drug supervision and the limit of drug supervision resources. According to the CFDA, there were 195,523 medicine labels in China in 2007, including biological, chemical, and traditional Chinese medicines. Given the rapid advances in pharmaceutical sciences and technologies, new and innovative medicines have been introduced to the drug market at an accelerating pace. In addition, the demand for each medicine is high in China given the country's huge population. So the efficient and effective supervision of drug distribution has become a significant public health issue to address 
in China. To this end, many innovative methods for drug supervision have been developed, e.g., the complaint inspection mechanism, random inspection mechanism, and electronic monitoring based on emerging IT (RFID, barcode etc), among others. Different drug supervision methods require different resources, incur different costs, and have different supervision outcomes. However, there is no theoretical analysis on which supervision methods should be adopted for some drugs. Drug-related incidents continue to occur in China despite the large investment of supervision resources by the government. So the contemporary challenge facing the CFDA is to devise and deploy proper supervision approaches and modes for different drugs so that the limited supervision resources are efficiently used to effectively supervise drug distribution without making any adverse effect on patients and society.

The nature of different drugs differs markedly. Some drugs are highly profitable, and the probabilities of faking and making inferior-quality versions of such drugs are high, while others are marginally profit and no one bothers to counterfeit them. Some drugs have serious harmful effects on patients and society if their quality does not comply with the standards or the drugs are abused, while others have little impact on patients and society. Evidently, the CFDA should focus its limited supervision resources on the important drugs. Against this backdrop, we propose that drugs be classified from the perspective of drug supervision and we identify a proper supervision approach for each class of drugs in this paper.

\section{Method}

\subsection{Drug Classification}

At present, there are classification methods for drugs for different purposes. In order to ensure drug safety for patient treatment in China, drugs are classified into prescription and non-prescription drugs as stipulated in Classification Management Method of Prescription and Non-prescription Drugs (trial) enacted in 2000. Patients can buy non-prescription drugs freely, while the sale and use of prescription drugs must be under the direction of doctors ${ }^{4}$. At the retail level, pharmacies adopt different classification methods for the purposes of search and revenue management. Some hospital pharmacies use the ABC classification based on drugs' prices and usage volumes. However, there is no drug classification method for the purpose of drug supervision.

As discussed above, it is evident that greater supervision efforts should be devoted to drugs that are very harmful to patients and society should they be faked or produced in inferior quality, and drugs with high probabilities of being faked. The harmfulness of drugs is related to their safety requirements, while the probabilities that drugs are faked or produced in low quality are related to the faked drugs' profits to the fakers (economic values). In view of this, we propose a classification method for drugs based on drugs' safety requirements and economic values for the purpose of efficient and effective supervision of drug distribution.

The safety requirements of a drug defined in the Pharmaceutical Administration Law are stipulated from the perspective of medical use of the drug and refer to the degree of humans' adverse reaction after using the drug. In contrast, in this paper we define the safety requirements of a drug from the perspective of drug distribution supervision, which means the degree of harm caused to the patient when a fake or inferior-quality drug is used or the degree of harm caused to humans or society when a drug is out of control or abused. The higher the harm is, the higher are the safety requirements for a drug. For example, a medicine treating heart attacks should have higher safety requirements than a medicine treating common cold because a patient with a heart attack will have very serious consequences if he/she takes a fake or inferior-quality drug. Another example is that a medicine with poison or strong side effects (e.g., antipsychotic drugs) should have higher safety requirements than a general medicine because there will be a big threat to humans or society if such a drug is out of control or abused. We define drug safety requirements in the above way because the main aims of drug distribution supervision are to alleviate the harm of fake and inferior-quality drugs to patients and to prevent someone from using harmful drugs to endanger humans and society. It follows that the CFDA should pay great attention to drugs with high safety 
requirements to keep patients and society safe.

In order to determine the safety requirements of a particular drug, many factors need to be taken into consideration. Table 1 summarizes the factors that affect the safety requirements of a drug. Generally speaking, drugs having strong pharmacological effects and more adverse reactions, being more easy to produce abusive addiction or bacteria resistance, and being used under the direction of physicians should have higher safety requirements, e.g., narcotic drugs, radioactive drugs, psychotropic drugs, toxic drugs, and drugs used to cure sudden serious illnesses such as heart attacks, cerebral hemorrhage, and so on. Drugs that are mainly used to treat common diseases and has less adverse effect on society should have lower safety requirements.

Table 1. Factors that affect the safety requirements of drugs

\begin{tabular}{|c|c|c|}
\hline & $\begin{array}{l}\text { Drugs with high } \\
\text { safety requirements }\end{array}$ & $\begin{array}{l}\text { Drugs with low safety } \\
\text { requirements }\end{array}$ \\
\hline $\begin{array}{l}\text { The degree of harmfulness of the disease to } \\
\text { the patient's life }\end{array}$ & high & low \\
\hline Pharmacological effects & strong & weak \\
\hline Adverse drug reaction & more & less \\
\hline The effects of misuse and abuse & $\begin{array}{l}\text { easy to cause } \\
\text { addiction }\end{array}$ & not easy to cause addiction \\
\hline $\begin{array}{l}\text { Whether it is easy to produce drug } \\
\text { resistance for bacteria }\end{array}$ & yes & no \\
\hline Applicable symptoms & $\begin{array}{l}\text { under guidance of } \\
\text { physician }\end{array}$ & $\begin{array}{l}\text { can be used by patients } \\
\text { themselves }\end{array}$ \\
\hline The time of staying in the body & longer & shorter \\
\hline The aftermaths of clinical trials & $\begin{array}{l}\text { major safety } \\
\text { incidents occurring }\end{array}$ & $\begin{array}{l}\text { major safety incidents not } \\
\text { occurring }\end{array}$ \\
\hline $\begin{array}{l}\text { The gap between treatment dose and toxic } \\
\text { dose }\end{array}$ & small & big \\
\hline $\begin{array}{l}\text { Whether it is a special drug for the } \\
\text { treatment of some major diseases }\end{array}$ & yes & no \\
\hline Whether it contains ephedrine & yes & no \\
\hline $\begin{array}{l}\text { Whether it is used by pregnant women or } \\
\text { children }\end{array}$ & yes & no \\
\hline
\end{tabular}

It is evident that the motivation for faking or making inferior-quality drugs is profit making. Hence it is more likely that drugs with high profitability are faked. Because of this, we define the economic value of a drug as the drug's profitability to the offender that fakes it or makes low-quality versions of it. There are two factors that affect the economic value of a drug, namely price and sale. It is natural that drugs with high price are more likely to be faked. For example, we usually see faked versions of new and innovative medicines and valuable Chinese medicines in the market. On the other hand, some drugs commanding low prices but large sales are also likely to be faked. Table 2 lists the factors that affect the economic values of drugs.

Table 2. Factors that affect the economic values of drugs

\begin{tabular}{|l|l|l|}
\hline & $\begin{array}{l}\text { Drugs with high } \\
\text { economic values }\end{array}$ & $\begin{array}{l}\text { Drugs with low } \\
\text { economic values }\end{array}$ \\
\hline Price & high & low \\
\hline Demand & high & low \\
\hline
\end{tabular}

It should be noted that drugs' economic values can change under some conditions. For example, radix isatidis has a low price and low demand generally, so it is a drug with a low economic value. But its demand and price increased drastically in 2003 when SARS attacked China, rendering it a drug with a high economic value during this time. Similarly, some drugs with high economic values, e.g., new or innovative drugs, can become drugs with low economic values over time.

Summarizing the above discussion, we see that there are serious consequences to humans and society when some drug-related incidents occur for drugs with high safety requirements and that drugs with high economic values are more likely to be faked. So the CFDA should pay greater 
attention to such drugs to reduce the harm and probabilities of faking and making inferior-quality versions of them. To this end, we propose in Figure 1 a drug classification method for drug supervision purposes based on drugs' safety requirements and economic values, whereby drugs are classified into four types, namely safe and low value (SL) drugs, safe and high value (SH) drugs, risky and high value (RH) drugs, and risky and low value (RL) drugs.

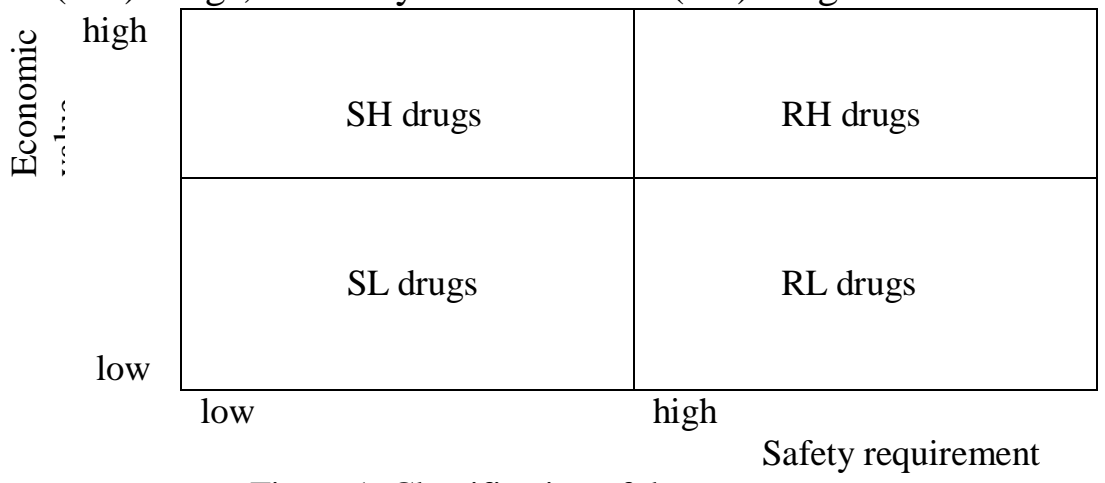

Figure 1. Classification of drugs

SL drugs have low safety requirements and low economic values. Iodophors, ethacriding lactate solution etc. are in this class. For this class of drugs, the probabilities of counterfeits are small, and the harm of fake and inferior-quality drugs to patients and society is small.

$\mathrm{SH}$ drugs have low safety requirements but high economic values. New drugs, valuable Chinese herbal medicine such as ganoderma, ginseng, cordyceps sinensis etc., and drugs with high demands belong to this class. For this class of drugs, the probabilities of counterfeits are high, but the harm of fake and low-quality drugs to patients and society is small.

RH drugs have high safety requirements and high economic values. Human serum albumin belongs to this class. For this class of drugs, the probabilities of counterfeits are high and fake or inferior-quality drugs may bring great harm to patients and society.

RL drugs' safety requirements are high but their economic values are low. Sleeping pills belong to this class. For drugs in this class, no one would care to fake them for economic reasons. The aim of supervising this class of drugs is to prevent these drugs from abuse and to ensure their legal use.

We should note that the classification of drugs for drug distribution supervision purposes is not fixed over time but should be dynamically adjusted according to changes in the economic and social environments. A drug usually belonging to one class may switch to another class as the environment changes. For example, honeysuckle bud and flower usually is an SL drug and almost no one would care to fake it. But during the SARS and Bird Flu onslaughts in China in 2003 and 2009, respectively, it became an SH drug and the genuine drug was difficult to acquire in the market.

\subsection{Classified Supervision}

At present, the laws that govern the supervision of drug distribution in China are the Pharmaceutical Administration Law of the People's Republic of China and Provisions for Supervision of Drug Distribution, which were promulgated by the CFDA in 2001 and 2006, respectively. Article 66 in the former states that "The pharmaceutical supervisory and administrative departments ...... shall regularly promulgate the results of sampling examinations and inspections on the quality of pharmaceuticals" "5]; Article 74 states that "Those who produce and sell fake medicine shall have his unlawfully produced or sold medicine and any unlawful income confiscated, and concurrently be fined a sum of money more than two but less than five times the value of the medicine unlawfully produced and sold" ${ }^{[5]}$; and Article 77 states that "Those who provide facilities including transportation, preservation and storage that have been or should have been aware of the fakery or inferior quality of the pharmaceuticals, shall have the entirety of their income from such transportation, preservation and storage confiscated, and concurrently be fined a sum of money more than $50 \%$ but less than three times the value of the unlawful income." ${ }^{[5]}$ In addition, Article 23 in The Eighth Criminal Amendment also refers to the supervision of drug distribution, which only stipulates that the offender must be fined by the government, but without stating how and how much to fine. In other words, if a person/firm that produces or sells fake drugs is caught by the 
government, the offender will be fined an amount based on the values of the fake drugs but not the degree of damage to patients and society. On the other hand, if the offender is not caught by the regulators, they will make economic gains without any consequences. Given that the government only adopts sampling examination, while the number of medicines is huge in China, the probability of catching a faker is small in reality. As a result, there have been a lot of fakery and inferior-quality drug cases in recent years.

In recent years, the CFDA has developed a host of methods for drug supervision by using emerging information technologies (e.g., barcode, RFID, drug electronic monitoring system etc.). Drug electronic monitoring system was a hot topic in drug supervision. It works as follows ${ }^{[6]}$ : the CFDA issues a unique barcode to each unit of a drug before it enters the distribution process, and stores the barcode containing the relevant attributes of the drug in its database. For each transaction, the buyer and the seller have to log on the database to change the ownership of the drug. So the whole distribution process of each unit of a drug is recorded in the CFDA's database and consumers can track the entire process from production to distribution to sales via computer terminals. Evidently, given such a strict method of drug supervision, fake or inferior-quality drugs have no chance to enter the drug market. However, given the vast number of drugs and the corresponding gigantic number of barcodes to store, the CDFA's database is undoubtedly very huge and complex. Since the sellers and the buyers have to log on the drug supervision website to change their drugs' attributes for each transaction, the database query and search are very slow. This renders the drug distribution process highly inefficient. Hence it is common to see that a large number of medicines stacked outside drug stores awaiting changes of their attributes, which gives rise to many problems and causes lots of complaints by drug distributors. As a result, the drug electronic supervision system as of now can only be used to supervise a few kinds of drugs that have low demands and high safety requirements.

The CFDA has issued a notice to suspend the implementation of the relevant provisions of the drug electronic monitoring system, and to solicit opinions from the public on the "quality management regulations for drug administration” (revised draft). So far, the drug electronic monitoring system has been halted for 8 years. It can be seen from the "quality management regulations for drug administration" (revised draft) ${ }^{[7]}$ that the drug traceability system will replace the electronic regulatory code and assume the responsibility of drug supervision.

In the previous section, we classified drugs into four classes based on their safety requirements and economic values. In addition, the probabilities and the harm of fake and inferior-quality drugs in different classes are different. The Chinese government cannot attain the supervision aim if it applies the same supervision approach to all the drugs since the supervision resources are limited. To address this significant issue, we recommend that the government adopts different supervision approaches for different classes of drugs. We put forward two supervision strategies, namely Punishing Once Detected (POD) and Process Monitoring (PM).

The POD strategy, just as stated in the provisions of the Pharmaceutical Administration Law of the People's Republic of China and Provisions for Supervision of Drug Distribution, means that drug producers, distributors, and sellers are inspected and will be punished according to the above two laws once they are caught committing drug-related offences. This strategy is simple, incurs a low supervision cost, and needs no supervision of the entire drug distribution process spanning production, distribution, and sale. So drug distribution is efficient under POD. However, there are chances that fake and inferior-quality drugs enter the market and will not be found under this strategy. Of course, the more frequent inspections are performed, the higher is the inspection cost, the higher is the probability of catching the offenders, and the less are the probabilities that fake or low-quality drugs are made.

The PM strategy is to use some technology (e.g., RFID, electronic supervision, tracking the whole process etc.) to monitor the entire drug distribution process to prevent fake and inferior-quality drugs from entering the market. This strategy can reduce the probability of drug-related incidents, and ensure the safety and reliability of drug distribution. However, this 
strategy will incur a high logistics cost and result in low logistics efficiency, e.g., the case of electronic supervision discussed above. In addition, the PM strategy can be deployed in increasing stringent forms as follows: total volume supervision, drug batch supervision, drug unit supervision etc. The stricter the form is, the less is the possibility that fake or low-quality drugs can enter the market, the higher is the logistics cost, and the lower is the logistics efficiency.

Summarizing the above discussions, we see that the POD and PM strategies have their own merits and shortcomings, while they are complementary to each other. We recommend that a proper combination of the two strategies be adopted to supervise different classes of drugs. Table 3 illustrates different combinations of the two drug supervision strategies for different types of drugs.

Table 3. Supervision approaches for different types of drugs

\begin{tabular}{|l|l|}
\hline Drug type & Supervision approach \\
\hline SL drugs & POD \\
\hline SH drugs & Strong POD with PM as a supplement \\
\hline RH drugs & Strict PM (e.g., unit PM) \\
\hline RL drugs & PM with POD as an auxiliary \\
\hline
\end{tabular}

In general, POD is used to supervise drugs with low safety requirements to increase the efficiency of distribution, while PM is used to supervise drugs with high safety requirements to reduce the probabilities that fake or inferior-quality drugs are made and enter the market.

For SL drugs, which have low economic values and cause little harm to patients and society, POD can achieve a good supervision effect to prevent their faking. For this class of drugs, it is apposite to adopt the supervision methods specified in the laws Pharmaceutical Administration Law of the People's Republic of China and Provisions for Supervision of Drug Distribution. That is why there are few fake and inferior-quality drugs of this type in China.

For SH drugs, their high economic values lead to high probabilities to fake and make inferior-quality drugs. However, the harm of such fake and inferior-quality drugs to patients and society is not very large. For this class of drugs, we can mainly use POM with higher inspection frequencies and higher penalties on the offenders. Of course, we can use the PM strategy as a supplementary approach without affecting the efficiency of drug distribution and increasing the logistics cost. The total volume supervision or drug batch supervision is a suitable form of PM to apply. Therefore, for RH drugs, it is viable to adopt strong POD supplemented with a loose form of PM.

RH drugs have high economic values and high safety requirements. So the probabilities of faking the drugs are high, and fake and inferior-quality RH drugs bring great threats to patients and society. In order to prevent fake and inferior-quality drugs from entering the market, we should adopt the strictest form of the PM strategy (e.g., electronic drug monitoring discussed above) to reduce the probabilities of faking and making inferior-quality drugs to zero.

For RL drugs, the probabilities of faking or making inferior-quality drugs are small because of their low profitability. The aim of supervising this class of drugs is to keep their use under control and to prevent them from abuse because they are harmful to humans and society if they are abused. So we should use a relatively strict form of the PM strategy to reduce the chances of illegal access to such drugs. Once someone caught possessing such drugs unlawfully, they should be fined heavily. The penalty should be set based on the harm to humans and society rather than the values of the drugs. By using mainly a relatively strict form of PM and using POD as an auxiliary supervision approach, we can keep the production, distribution, sale, and usage of RL drugs under control.

In order to make the best use of the limited supervision resources to achieve a better supervision effect, we propose that drugs be classified based on their safety requirements and economic values, and that a proper supervision approach be applied for each class of drugs. Of course, the classification of drugs and the corresponding supervision approaches are not static. In other words, in view of changes in society, economy, and technology, the classification of drugs for drug distribution supervision purposes should change with corresponding changes in the drug supervision approaches. For example, radix isatidis is an SL drug and the POD strategy should be applied to supervise it generally. However, it became an RH drug during the period of SARS and 
the PM strategy should be used as a complementary supervision approach.

\section{Results and Conclusions}

Because of the tussle between a large number of drugs and limit supervision resources, frequent occurrences of incidents related to fake and inferior-quality drugs have become a serious public health issue in China. We find that the main culprit is the supervision methods adopted by the CFDA. To adequately address this issue, we propose a new classification of drugs based on drugs' safety requirements and economic values, two key factors in drug distribution supervision. We also propose a proper supervision method for each class to help the government to efficiently use the supervision resources to effectively supervise drug distribution in China.

\section{Discussions}

At present, hospitals and drugstores account for $80 \%$ and $20 \%$ of drug sales, respectively, in the Chinese pharmaceutical market. It is relatively easy to supervise drug distribution for drugs sold through hospitals. However, selling drugs through hospitals gives rise to a host of problems, e.g., over-treatment of patients and incurring high medical costs to patients. In order to overcome these problems and sever the profit links between doctors and pharmacies, the Chinese government has promulgated the policy of separation of prescribing and dispensing of drugs $^{[8]}$. This policy will lead most patients to buy medicine from drugstores. Incidentally, with the development of e-commerce, an increasing number of firms sell medicines via the Internet. This will greatly facilitate patients to buy medicines. Due to the difficulty of supervising drug distribution, very few firms are granted licences by the government to sell drugs via e-commerce channels. Patients can only purchase SL drugs from drugstores and the Internet. So the CDFA should properly classify drugs and adopt appropriate combinations of the POD and PM strategies to efficiently and effectively supervise each class of drugs to ensure that patients can safely and conveniently buy and use the drugs they need at reasonable costs. This is very important for addressing a significant public health issue in China.

\section{Acknowledgement}

The authors sincerely thank to the International Center for Informatics Research, the anonymous reviewer. This work was supported by Beijing Logistics Informatics Research Base, the National Natural Science Foundation of China (Nos. 71390334 and 71132008), and the Program of New Century Excellent Talents in University (NCET-13-0660).

\section{References}

[1] “2015 CFDA Annual Report”, http://www.sfda.gov.cn/WS01/CL0108/143640.html

[2] “2016 CFDA Annual Report”, http://www.sfda.gov.cn/WS01/CL0108/172895.html

[3] Guangming Online, http://health.gmw.cn/2014-09/03/content_13006745.htm

[4] "Classification Management Method of Prescription and Non-prescription Drugs", enacted by the CDFA, 2000

[5] "Pharmaceutical Administration Law of the People's Republic of China", promulgated by the CDFA, 2001

[6] http://www.drugadmin.com/

[7] "quality management regulations for drug administration" (revised draft), promulgated by the CFDA, 2016

[8] "Deepening the Reform of the Medical and Health Care System", promulgated by Third Plenary Session of the 18th Communist Party of China Central Committee, 2014, http://www.chinadaily.com.cn/m/chinahealth/2014-08/24/content_18476807.htm 